Rev. salud pública. 13 (6): 1031-1050, 2011

Ensayos/Essay

\title{
Aproximación a la construcción teórica de la promoción de la salud
}

\section{An approach to the theoretical construction of healthcare promotion}

Rocío Robledo-Martínez y Carlos A. Agudelo-Calderón

Instituto de Salud Pública. Facultad de Medicina. Universidad Nacional de Colombia. rrobledom@unal.edu.co caagudeloc@unal.edu.co

Recibido 23 Octubre 2011/Enviado para Modificación 17 Noviembre 2011/Aceptado 6 Diciembre 2011

\section{RESUMEN}

Se examinan las definiciones de promoción de la salud desde la teoría del conocimiento, en sus aspectos ontológicos y epistemológicos como son considerados por diversos paradigmas. Se utiliza una periodización en tres fases En la fase de antecedentes (1920-1973) se producen los primeros distanciamientos frente al paradigma positivista y la promoción de la salud hace parte de las definiciones de salud pública. En la fase fundacional (1974-1986) la promoción de la salud adquiere identidad propia y se transforma en políticas explícitas, en medio de escenarios de confluencia de paradigmas. En la fase de consolidación (19872008), se articulan ampliamente los paradigmas positivistas y no positivistas, extendiéndose a los aspectos metodológicos.

Palabras Clave: Promoción de la Salud, conocimiento, epistemología, salud pública (fuente: DeCS, BIREME).

\begin{abstract}
Definitions of healthcare promotion were examined from the theory of knowledge regarding its ontological and epistemological aspects as considered by several paradigms. Three-phase periodisation was used. The first distancing regarding the positivist paradigm and healthcare promotion through definitions of public health was made during the background phase (1920-1973). The promotion of healthcare acquired its own identity and became explicit policy in the midst of scenarios involving the confluence of paradigms during the foundational phase (1974-1986). Positivist and non-positivist paradigms became broadly articulated during the consolidation phase (1987-2008), becoming extended to methodological aspects.
\end{abstract}

Key Words: Health promotion, knowledge, epistemology, public health (source: $\mathrm{MeSH}, \mathrm{NLM}$ ). 
E n el presente ensayo se intenta una aproximación a los conceptos de promoción de la salud-PS, como han sido construidos en momentos clave de su historia. Con este propósito se utilizarán exclusivamente las definiciones sobre la PS que tienen alcance de hitos conceptuales o paradigmáticos. Esto, por cuanto numerosas definiciones o enfoques de PS están centrados en las metas de la salud pública o en los medios para lograr resultados (1).

El marco teórico y metodológico incluye los siguientes aspectos:

Se abordan las definiciones de PS desde la dimensión de la teoría del conocimiento (Gnoseología) y, por tanto, como resultado de la relación (no excluyente) entre el sujeto (Ser cognoscente) y el objeto, y desde la dimensión de investigación. En este sentido, se consideran las aproximaciones conceptuales a la PS desde el punto de vista ontológico (¿Cuál es la naturaleza de lo conocible, cuál es la naturaleza de la realidad?) y epistemológico (¿Cuál es la naturaleza de la relación entre el investigador y lo conocible?).

En teoría de conocimiento diversas posiciones filosóficas son reconocidas. Con respecto a la fuente u origen del conocimiento: racionalismo, empirismo, intelectualismo y apriorismo; con respecto al conocimiento posible, se mencionan el dogmatismo, el escepticismo, el pragmatismo y el criticismo.

Se intenta rastrear los paradigmas que pueden subyacer en las definiciones de PS. Entre los numerosos modelos existentes, se utilizó el de Guba y Lincoln (2) entendiendo por paradigma un "...sistema básico de creencias o modo de ver el mundo que guía al investigador, no sólo en elecciones del método sino en caminos epistemológicos y ontológicos fundamentales." En consecuencia, cada paradigma se ubica de una manera diferente frente a la teoría del conocimiento y sus posiciones filosóficas. Estos autores consideran cuatro paradigmas: positivismo, postpositivismo, teoría crítica y constructivismo. Examinar estos paradigmas va más allá que el alcance del ensayo.

Cabe mencionar las dificultades y limitaciones del abordaje del ensayo: la relación entre la respuesta ontológica y epistemológica es necesaria, pero en las definiciones de PS con frecuencia no es explícita; las categorías 
con que identifican paradigmas son numerosas y muchas de ellas son posteriores a las definiciones de PS, por lo cual con frecuencia utilizamos analogías; en las disciplinas y profesiones conviven diversas corrientes de pensamiento en términos paradigmáticos y metodológicos y es frecuente la migración y combinación de uno y otro; la salud pública y la PS son un campo científico y profesional en el cual confluyen diversos saberes, agregándole un ingrediente adicional de dificultad al abordaje ontológico y epistemológico.

En todo caso, se espera aportar a la interpretación del papel de la PS y su aplicación, al tiempo como herramienta de desarrollo social y de transformación de los sistemas de salud $(3,4)$.

Desde el punto de vista teórico y conceptual se ha considerado que la PS tiene unos antecedentes lejanos, una fase fundacional y un proceso de consolidación más reciente que ha transitado tan sólo durante un cuarto de siglo de tensiones, experiencias y resultados (5). En cada una de las fases se intenta hacer una ubicación de contexto, una aproximación a las definiciones de PS y unos comentarios sobre los trayectos ontológicosepistemológicos.

Fase de antecedentes (1920-1973)

Un contexto de transformaciones globales

Una fase de más de cincuenta años no es sólo larga sino que ésta en particular representa en el mundo occidental la consolidación de la época moderna -iniciada tres siglos atrás- en sus esquemas básicos de organización y desenvolvimiento.

A continuación se reseñan algunos de los aspectos clave que consolidaron la época moderna en el Siglo XX, incluyendo aquellas que tienen una relación más directa con el tema de reflexión.

- En el largo período considerado (1920-1973) el mundo experimentó transformaciones demográficas, sociales y políticas profundas y complejas. Gran parte de los países desarrollados completaron la transición demográfica y los países en desarrollo avanzaron en la misma. Luego de dos guerras 
mundiales, se produjo una configuración geopolítica e internacional como mundo bipolar, en el marco del equilibrio nuclear estratégico y la multipolaridad política. Los modelos de desarrollo económico comenzaron a superar el esquema heredado de la revolución industrial, se consolidó el comercio internacional y el capitalismo mundial dio un salto en la organización con base en la irrupción de las corporaciones transnacionales, como centros del poder económico mundial.

- Entre la crisis económica de los 30 y la crisis del petróleo de los 70, en los países capitalistas desarrollados, las relaciones políticas internas renovadoras llevaron a Estados de Bienestar que elevaron progresivamente el nivel de vida de la población, ampliaron el gasto público, los programas sociales y los correspondientes aparatos de Estado.

- Por décadas enteras se llegó al predominio de la teoría morbicentrista centrada en el manejo de la enfermedad, con énfasis en la atención hospitalaria, la tecnología y los medicamentos. Así mismo se configuró una visión preventiva subordinada a la práctica médica que, a su vez, redujo la promoción a la educación en salud de los individuos, a cargo de la misma institución dedicada a atender enfermos.

- La salud pública hizo parte de las tendencias anteriores, pero al tiempo gestó procesos propios. Creció con base en los aportes de saberes diversos que apuntaban a la salud de la población y los individuos (ciencias básicas, ciencias sociales, administración, epidemiología, etc.), las tecnologías disponibles y en desarrollo, y la adopción e implementación de políticas públicas y programas que incidieron de manera dramática en la esperanza de vida de la población.

- Por otra parte, las políticas públicas también se ocuparon de la creación y posterior transformación de los sistemas de salud, especialmente en los países desarrollados.

\section{Aproximaciones iniciales}

Tempranamente, en momentos en los cuales los sistemas de salud modernos daban sus primeros pasos como sistemas nacionales, las preocupaciones de algunos pensadores se concentraron en darle fundamento conceptual 
a la salud pública. Es por ello que los aportes en PS hacen parte del proceso de estructuración conceptual de la salud pública, como sistema de pensamiento, política y acción. Los aportes iniciales fueron de Winslow (6) y Sigerist (7).

En la reconocida definición de Winslow de salud pública se encuentra una aproximación a la promoción de la salud. Entresacada de aquella definición, la promoción de la salud correspondería al “... esfuerzo de la comunidad dirigido a... la educación de los individuos en los principios de la higiene personal... y el desarrollo de la maquinaria social para asegurar a cada individuo en la comunidad un estándar de vida adecuado para el mantenimiento de la salud" (8). Pese a su formación positivista, Winslow adopta una definición de salud pública en una perspectiva de causación amplia y holística. En respuesta a los retos que afrontaba en su momento se remite a los determinantes de la salud y otros aspectos complementarios.

Por su parte, Sigerist se refería a la promoción en términos de las condiciones que determinan la salud: “...proporcionando un nivel de vida decente, buenas condiciones de trabajo, educación, cultura física, medios de descanso y recreación" (9). A propósito de esta definición es necesario recordar que pese al predominio en su momento de los abordajes positivistas de la historia, Sigerist logra en gran parte de su obra estructurar un enfoque más interpretativo.

Si bien el enfoque de ambos pensadores es similar, las motivaciones de uno y otro son diferentes. Aunque ambas definiciones tienen en común los determinantes de la salud, los conceptos utilizados por Winslow remiten a las categorías de educación, individuo, grupos sociales y sociedad, mantenimiento y mejoramiento de la salud. Así mismo, es evidente que mientras Winslow pone en relación las diferentes dimensiones conceptuales, Sigerist hace énfasis en los determinantes.

Desde su formulación estas definiciones incidieron en las construcciones conceptuales de la salud pública y de la PS y dieron lugar a maneras de comprender la naturaleza de la PS y la relación entre la investigación y lo conocible de la PS. Pero llama la atención la baja intensidad que durante décadas tuvo el desarrollo teórico y conceptual en ambos campos, quizás en parte inducido por el amplio predominio, como ya se ha planteado, de la concepción curativa o de atención médica y hospitalaria de la salud y su 
complemento preventivo. Sin embargo, debe destacarse que el predominio avasallante de la medicalización no impidió que se conservara y luego renaciera, en algunos círculos y grupos de analistas y decisores, el interés por la salud positiva, con base en la evidencia proporcionada durante años por las ciencias sociales y la epidemiología, en términos del papel de los determinantes de la salud, la calidad de vida y las desigualdades (10). Cabe mencionar que desde la década de los 50 se consolidó la Sociología médica (o Sociología de la Medicina) en Europa y Estados Unidos, proporcionado interpretaciones sobre la salud y la enfermedad, tanto desde las vertientes tradicionales positivistas como desde corrientes estructuralistas (11).

Aunque tales expresiones alternativas a la corriente principal, no modificaron la discontinuidad en los momentos de las formulaciones conceptuales, característica de la fase de antecedentes, contribuyeron, de la década de los 50 en adelante, al surgimiento de nuevas aproximaciones estructuradas sobre la salud, la salud pública y la PS, justamente cuando comenzaba a ser evidente que las tendencias y realidades universalistas de los sistemas de salud, si bien contribuían a la calidad de vida, no podía afirmarse lo mismo con respecto a la esperanza de vida, y los crecientes costos de aquellos no correspondían con los resultados. En estas condiciones, resurgieron enfoques de salud pública y de PS centrados en los determinantes de la salud, que se expresaron en dos propuestas. McKweon fundamentó la promoción de la salud desde el punto de vista histórico (1214) al documentar el papel del crecimiento económico, el incremento del estándar de vida y el mejoramiento de la nutrición como fuentes primarias de la salud de países desarrolladas. Las interpretaciones de la salud, la salud pública y la PS de McKeown fueron dominantes en Europa de 1950 a 1970.

Laframboise construyó un marco conceptual del campo de salud, buscando una definición apropiada que facilitara la formulación y aplicación de políticas públicas de salud y salud pública (10). El campo de salud consideraba el estilo de vida, el ambiente, la organización de la atención, la biología humana y su aplicación clínica, convirtiendo la salud en un problema de la sociedad y no sólo del sector salud. Cada uno de estos conceptos fue planteado de manera amplia; el esquema del campo ordena y pone en relación algunos de los determinantes clave de la salud y resultaba útil desde el punto de vista político e instrumental. Pero el mismo esquema genera un horizonte teórico y conceptual que representa un sistema 
relativamente cerrado. En medio de estas ventajas y limitaciones, el campo de salud apunta también a la PS. El propio Laframboise lo sugiere: "El reto en el campo de la salud en Canadá es mantener el presente alto nivel de cuidado de salud e investigación médica, mientras concentramos nuestros esfuerzos para llevar a un nivel similar las áreas de estilo de vida y medio ambiente..." (15).

\section{Distanciamientos ontológicos}

Debe destacarse que la fase de antecedentes como proceso de construcción de una teoría moderna de la salud pública, representa el esfuerzo de unos pensadores que encararon, en palabras de Porter (16), grandes narrativas de progreso, con base en el avance tecnológico de la ciencia, la medicina y la salud pública, en términos de la reducción masiva de la mortalidad en los países desarrollados. Pero, al tiempo, representa tan sólo unos primeros aportes seminales, decisivos para el futuro inmediato de la salud pública y la PS. Apoyándose ampliamente en los conocimientos y evidencias generadas por disciplinas positivistas, los pensadores comentados construyeron, desde las disciplinas de la administración, la historia, la sociología y la política, un cuerpo conceptual de salud pública y PS, que permitió un primer distanciamiento ontológico y epistemológico, con respecto a la corriente principal positivista. Las definiciones de PS corresponden al abordaje de la realidad como un fenómeno relativo y una manera de conocerla, que sugieren fuertemente un paradigma no positivista. Si estas definiciones fuesen de hoy, cabrían sin tensiones, siguiendo a Guba (2) en los paradigmas de la teoría crítica y el construccionismo.

Fase fundacional (1974-1986)

\section{Contexto de crisis}

Se reseñan únicamente tres grandes tendencias.

- El acentuamiento de la crisis económica en los años 70, aunada al incremento histórico del gasto público, debilitaron en los países europeos las configuraciones políticas internas dominantes en la fase previa, y progresivamente viraron a opciones de gobierno conservadores $\mathrm{y}$ posteriormente neoliberales.

- El desarrollo de los sistemas de salud, desde el enfoque morbicentrista, comenzó a presentar costos crecientes, ocasionados por la provisión de servicios basada en la hospitalización, las tecnologías y los medicamentos. 
Esta tendencia incrementó aún más el gasto público sin aportar mejoramientos adicionales en la esperanza de vida.

- Las dinámicas de movimientos ciudadanos heterogéneos, fortalecieron la participación social y las democracias locales, expresando un creciente empoderamiento y deseos de ejercer un mayor control sobre sus vidas.

Diversidad y política general de PS

A partir de 1974 toma forma un proceso que lleva, en medio de continuidades y fragmentaciones conceptuales, a una política general de PS.

Un hito justifica denominar esta fase como fundacional: la PS adquiere el estatus de política pública nacional. En el marco de un trabajo institucional, Lalonde, apoyándose de manera explícita en McKeown y Laframboise, adopta el concepto de campo de salud y lo utiliza como fundamento de una política pública nacional de salud, para Canadá (17). La salud pública adquiere una dimensión de política de Estado y de sociedad y se convierte en un propósito nacional, superando la utilización puramente instrumental por parte del Estado, como había ocurrido en la fase de antecedentes. Por otra parte, la PS adquiere la misma dimensión anterior y se individualiza como un aspecto central de la política de salud pública. Lalonde afirma: "El sistema de atención, sin embargo, es sólo uno de los muchos caminos para mantener y mejorar la salud. De importancia igual o mayor para incrementar el número de días libres de enfermedad en la vida de los canadienses ha sido el incremento del estándar de vida general, las importantes medidas sanitarias para proteger la salud pública y los avances en la ciencia médica” (17).

Debe señalarse que el Informe Lalonde, incorpora como parte de la política nacional, la prevención de enfermedades en todo su alcance (análisis de los riesgos individuales y poblacionales, y estrategias a poner en práctica). El propósito es también explícito: acrecentar la viabilidad de la política de salud y atenuar la subordinación de la prevención al enfoque curativo. Es notorio que, de allí en adelante, la gran mayoría de políticas nacionales de salud y de promoción adoptan este esquema. Sin embargo, con alguna frecuencia se plantea que este esquema debilita la promoción o, cuanto menos, induce confusión (18). 
Pocos años después del Informe Lalonde, la percepción sobre los avances y resultados de la nueva política, era más bien pesimista. En su momento, Buck planteó que las recomendaciones no se habían llevado a la práctica, principalmente por las debilidades con respecto al elemento medio ambiente que sería determinante para los demás elementos del campo de salud (19).

Pero aquella no fue la única interpretación que expresaba desazón ante los escasos resultados de la política de salud canadiense, especialmente frente a las expectativas de corto plazo. De hecho, surgieron varias propuestas y con consecuencias diversas. Cabe mencionar el enfoque planteado por Blum (20), ampliamente difundido y utilizado en países de América Latina. Esta propuesta desarrolla el modelo de campo de salud en un sentido marcadamente sistémico y cuantitativo, por el cual pasa su aplicación en administración y política.

Por su parte, el Departamento de Salud de los Estados Unidos presentó en 1979 el informe "Gente Sana", construido siguiendo el esquema del Informe Lalonde, sobre la promoción de la salud y la prevención de la enfermedad. Se centra en los estilos de vida y la protección frente a riesgos (21).

Otras propuestas tomaron caminos diferentes

Antonovsky construyó un esquema explicativo de la adaptación al estrés y la adversidad. Se ocupó de la disposición personal que puede generar resiliencia o capacidad de adaptación ante los estresores de la vida diaria, basándose en los "recursos de resistencia generalizada" y en el "sentido de coherencia” (22). Este enfoque lo denominó con el término "salutogénico" y lo interpretó como fundamento de la PS: "A salutogenic orientation, then, as the basis for health promotion, directs both research and action efforts to encompass all persons, wherever they are on the continuum, and to focus on salutary factors" (23). El autor sostenía que el modelo salutogénico era aplicable al individuo, los grupos y la sociedad. Sin embargo, la construcción conceptual y metodológica se da en torno al individuo.

Por otra parte, resurgió el enfoque educativo como elemento central de la PS. Green consideró la PS como “...cualquier combinación de educación de la 
salud y apoyos relacionados de organización, políticos y económicos para la conducta que conduce a la salud" (24- 27).

En los años 1984 a 1986, la OMS se aproximó de manera explícita a una versión de la PS centrada en la capacidad de la gente para controlar los determinantes y los recursos personales y materiales disponibles (28). Como parte de este proceso institucional Nutbeam produjo un glosario de definiciones que identifica la PS como "El proceso para permitir a la gente que aumente el control sobre su salud y de ese modo mejore su salud" (29). El trabajo de la OMS sirvió de base documental para la Conferencia de Ottawa. En esta última, Epp presentó, en su discurso inaugural (30), una propuesta curiosa de PS que si bien parte de la misma definición de PS de la OMS, la concibe como un conjunto de mecanismos y estrategias de PS para afrontar los retos de la sociedad canadiense.

La Carta de Ottawa acogió la definición de PS construida por la OMS y la enriqueció. Es explícita al respecto: "La promoción de la salud consiste en proporcionar a los pueblos los medios necesarios para mejorar su salud y ejercer un mayor control sobre la misma. Para alcanzar un estado adecuado de bienestar físico, mental y social un individuo o grupo debe ser capaz de identificar y realizar sus aspiraciones, de satisfacer sus necesidades y de cambiar o adaptarse al medio ambiente. La salud se percibe pues, no como el objetivo, sino como la fuente de riqueza de la vida cotidiana. Se trata por tanto de un concepto positivo que acentúa los recursos sociales y personales así como las aptitudes físicas" (31). Adicionalmente, la Carta de Ottawa plantea determinantes en la forma de una lista específica de requisitos de la salud, subordinados a las capacidades.

El proceso adelantado por la OMS, la Conferencia de Ottawa y la Carta de Ottawa, produjeron un giro significativo en la construcción conceptual de la PS con respecto al Informe Lalonde:

- El esquema sistémico cerrado del campo de la salud es reemplazado por un esquema abierto de determinantes de la salud, que se interpretan como requisitos de la salud.

- La salud no es, en sí misma, un objetivo, sino, por una parte, un recurso y fuente de riqueza de la vida cotidiana y, por otra parte, resultado de los medios y capacidades de los "pueblos".

- El supuesto básico de la PS es “...proporcionar medios a los pueblos”. Esto deja de lado el supuesto de los enfoques que conciben la salud como 
un objetivo o resultado de las políticas operando sobre los determinantes o los estilos de vida.

\section{Confluencia de paradigmas}

La fase fundacional condensa los resultados de procesos económicos, sociales y culturales de distinta naturaleza. Se inicia con un modelo teórico estructurado -el campo de salud- y luego se abre a corrientes de pensamiento diferentes. Posteriormente, el proceso es canalizado por la OMS y da lugar a una definición de PS con carácter de política general.

En esta fase, una parte significativa de la construcción conceptual de la PS corresponde a procesos de orden grupal e institucional (Ministerio de Salud y Bienestar de Canadá, Departamento de Salud de los Estados Unidos y la OMS), lo cual facilitó la participación de diversas disciplinas y profesiones. Las que confluyeron en las definiciones de PS, incluyendo las de origen individual, fueron principalmente la salud pública, la política, la sociología, la epidemiología y la administración.

Desde el punto de vista de las definiciones de PS debe destacarse la ampliación creciente del enfoque de la salud pública desde los comportamientos individuales y los factores de riesgo a los problemáticas de nivel poblacional.

Por su parte, la Carta de Ottawa adopta una perspectiva ontológica y epistemológica que pasa por los medios y las capacidades personales y sociales para incidir en los determinantes, mientras que estos últimos son un horizonte de condiciones y no el centro gestor de la PS. Así mismo, pone en relación el empoderamiento y los recursos - materiales y simbólicos. Los aspectos inherentes al empoderamiento (políticas públicas, ambientes favorables, aptitudes personales, acción comunitaria, reorientación de los servicios sanitarios) se transforman en estrategias (31). En consecuencia, si bien el proceso en esta fase lleva a un escenario de confluencia de paradigmas, la definición de PS apunta más claramente a los paradigmas de tipo interpretativo, no positivistas. 


\section{Fase de Consolidación (1987-2008)}

\section{Contexto de globalización}

A finales de los 80 se producen en el mundo un conjunto de transformaciones profundas que dan lugar a la globalización. Debido al hundimiento de la Unión Soviética, pronto se configura un mundo unipolar caracterizado por el monopolio nuclear, la multipolaridad de bloques y la fusión corporativa supranacional. El mercado internacional adquiere dimensión mundial, se imponen las políticas de desarrollo con enfoque neoliberal, los Estados, en los países en desarrollo, son objeto de políticas restrictivas y los sistemas de salud entran en ciclos de reforma para adecuarlos a condiciones de mercado. Algunas de las consecuencias de la globalización que afectaron las condiciones laborales, la cultura y la urdimbre social de las comunidades y, por tanto, marcaron el proceso de desarrollo de la PS, fueron las siguientes: exacerbación de la pobreza y las desigualdades, nuevas formas de consumo y comunicación, cambios económicos, sociales, demográficos, ambientales y en la urbanización, rápidos y con frecuencia adversos.

\section{Las grandes orientaciones de PS}

La Carta de Ottawa apuntó a redefinir la PS y darle un sentido de política general. Pero la definición resultante no se tradujo en corriente principal, ni unificó las escuelas de pensamiento. En realidad, de allí en adelante, se produjeron reagrupamientos de los enfoques conceptuales centrados en los determinantes, el empoderamiento social y la educación, y se abrieron nuevos caminos que entrecruzaron, en un esquema de relaciones horizontales, los trayectos ontológicos y epistemológicos. Una dificultad adicional proviene del avance de las posiciones que se ocupan con insistencia creciente en los escenarios, estrategias y métodos de la PS, jalonadas por la experiencia de ciudades sanas y municipios saludables y, la multiplicidad de experiencias locales e institucionales de PS.

En esta fase es posible identificar por lo menos cuatro caminos de desarrollo conceptual.

El primero de ellos se desprende del enfoque central de empoderamientodeterminantes planteado en la Carta de Ottawa cuya línea de continuidad 
más explícita se encuentra en la experiencia de "ciudades sanas". Así mismo, se inscriben en esta misma tendencia una gran parte de las experiencias locales de PS adelantadas en muchos países, cuyo ímpetu y frecuencia decae, sin desaparecer, hacia el año 2000. Las experiencias de PS, ciudadanas y de ciudades, fueron espacios y momentos de creatividad que pusieron de presente enormes retos por resolver.

El segundo camino fue el de las conferencias internacionales (32) que se convirtieron en escenarios en los que se examinaron múltiples experiencias y se contrastaban con los enfoques conceptuales o las valoraban como posibles políticas públicas. Estos lineamientos de campos de acción permitieron avanzar de manera sustancial en la identificación de escenarios, estrategias y métodos de la PS, válidos y factibles. La Conferencia de Adelaida, Australia, realizada en el año de1988, se centró en la importancia de la formulación de políticas públicas saludables; la Conferencia de Sundsval, Suecia, realizada en 1991, se enfocó en "Los Entornos Propicios para la Salud”; la Conferencia de Santa Fe de Bogotá, Colombia, realizada en 1992, planteó la interdependencia entre promoción de la salud y equidad; la Conferencia de Trinidad Tobago, realizada en 1993, reafirmó los principios y conceptos enunciados en la Carta de Ottawa; la Conferencia de Yakarta, en 1997, planteó lineamientos para la promoción de la salud en el siglo XXI: "Nuevos actores para una nueva era”; la V Conferencia realizada en México, D.F., en el 2000, declaró la equidad como elemento fundamental de la promoción de la Salud y formuló estrategias para la acción de la promoción de la Salud; la Conferencia que dio origen a la Carta de Bangkok para la Promoción de la Salud, de 2005, señaló: “el goce del más alto nivel alcanzable de salud, es uno de los más fundamentales derechos de cada ser humano sin distinción de raza, género, edad, religión, creencia política, condición social o económica”, y finalmente la Séptima Conferencia Mundial de Promoción de la Salud realizada en Nairobi, Kenya, en el año 2009, enfatizó en la importancia de utilizar varios procesos de participación, y emitió un "llamado a la acción”, por medio de la definición, ejecución de estrategias y compromisos, para implementar la salud a nivel mundial mediante la promoción de la salud.

Una interpretación aproximativa a los aportes y resultados de las Conferencias podría sugerir que permitieron avanzar, conservando en grandes líneas la perspectiva de la Carta de Ottawa, en la concepción y 
principalmente en los métodos y la instrumentalización de la PS, así como en el pulimento de lo que podría ser, en el futuro, una política mundial.

Un tercer camino puede ser identificado como aportes de las corrientes de pensamiento sobre la PS, de tipo individual o institucional, que se dan a lo largo de más de una década. Estos aportes tuvieron un marco exigente de retos que provenían de los movimientos de ciudades sanas y de las conferencias internacionales.

Cada uno de estos aportes se da desde sus propias bases teóricas y epistemológicas, pero hemos agrupado los autores para simplificar la presentación de las ideas centrales. Un primer grupo está conformado por cinco autores que proponen opciones de desarrollo instrumental de la PS o la reenfocan de manera unilateral en alguno de sus componentes.

Goodstadt planteó una aproximación instrumental: “A través de la instrumentación de programas, servicios y políticas efectivas el mantenimiento y mejoramiento de los niveles existentes de salud” (33).

Kar hizo lo propio desde la perspectiva de riesgos: “...y prevenir los riesgos de salud para lograr niveles óptimos en los determinantes de salud ambientales, sociales, de conducta y biomédicos el progreso del bienestar” (34).

O’Donnell desarrolló una aproximación cultural: "La ciencia y el arte de ayudar a la gente a escoger su estilo de vida para conducirlos hacia un estado de salud óptima" (35).

Green y Kreuter retomaron el enfoque educativo: “La combinación de apoyos educativos y del entorno para las acciones y condiciones de vida...que conduce a la salud” (36).

Un segundo grupo está conformado por tres autores que reafirman el papel de los determinantes de la salud. Labonté y Little: "Cualquier actividad o programa diseñado para mejorar las condiciones de vida social y ambiental de tal manera que aumente la experiencia de la gente acerca del bienestar" (37); Kickbusch: "PS no es el trabajo social de la medicina, por el cual puede conseguirse unos dólares extras. Realmente se trata de la nueva salud pública, un nuevo tipo de política pública basada en los determinantes de la salud"(38). 
El último camino se conforma con la nítida toma de distancia con respecto a los trayectos conceptuales previos. En 1990 la OPS aprueba la siguiente definición: "La PS es concebida, cada vez en mayor grado, como la suma de las acciones de la población, los servicios de salud, las autoridades sanitarias y otros sectores sociales y productivos, encaminados al desarrollo de mejores condiciones de salud individual y colectiva" (39).

Esta definición tiene como núcleo central la articulación de lo que hace la gente por su salud con las acciones del Estado y la sociedad. De allí resultó, en el lenguaje de técnicos y expertos, la coordinación intersectorial como un instrumento clave para lograr la PS. Este enfoque reorganiza las relaciones entre los componentes de empoderamiento, educación y determinantes, subordinándolos a la articulación y coordinación. Da la primacía a los expertos y los servicios de salud y, quizás por ello, fue adoptado por gran parte de los gobiernos y Ministerios de Salud de los países de América Latina. Adicionalmente, abrió el espacio para profundizar la relación asimétrica entre prevención y promoción, en manos de los servicios de salud que cuentan con los recursos para la atención y recuperación de los enfermos.

A partir del año 2002, las fronteras entre estos caminos pierden nitidez e incluso en algunos aspectos se diluyen. Por otra parte, los esfuerzos provenientes de diferentes corrientes de pensamiento y experiencias convergieron en los propósitos de desarrollar métodos válidos de investigación y en la producción de conocimiento nuevo, para responder los vacíos de conocimiento que impedían avanzar en la apropiación social e institucional de la PS. Más que los aspectos teóricos o paradigmáticos de la PS, a juzgar por los contenidos más frecuentes en las tres revistas internacionales que manejan la PS, se observa interés en la investigación en los aspectos metodológicos, los campos de la PS (Políticas, ambiente, estilos de vida, entornos comunitarios y servicios de salud), y escenarios (Ciudades y municipios saludables, ambientes físicos y de vida, trabajo y escuela).

Posteriormente el interés se extiende a la evaluación de la PS, desde los métodos, las experiencias y la investigación (40-43). Aunque desde su inicio los movimientos de ciudades sanas generaron métodos e indicadores de evaluación de resultados e impactos, sólo posteriormente se afronta la tarea de evaluar la promoción de la salud como enfoque de políticas, 
programas, estrategias y experiencias (44-45). Los enfoques y métodos de evaluación de la promoción de la salud están en proceso de construcción y ensayo. La evaluación de la PS es compleja y requiere la utilización de modelos y métodos diversos, provenientes de las ciencias sociales, la epidemiología, la ciencia política y la teoría de sistemas, entre otros (4647).

Más recientemente, el debate contemporáneo en PS está nuevamente en trasformación, en parte por las políticas mundiales de la OMS sobre los determinantes de la salud y la necesidad de los sistemas universales de salud $(48,49)$.

A manera de síntesis, a partir de la Carta de Ottawa, la PS entra en una fase de consolidación teórica y conceptual y al tiempo se expande en metodologías. Como movimiento social, político e institucional, desarrolla escenarios, métodos y estrategias, que se expresan en las Conferencias Internacionales y en diversas experiencias nacionales y locales (50-54).

Entre los propósitos de desarrollo de la PS, las políticas públicas ocupan un lugar de primer orden. En el ámbito global son escasos los países que cuentan con una política nacional y unitaria de PS. Sin embargo, en estos pocos países la influencia real sobre los sistemas de salud curativos es incipiente. Gran parte de los países cuentan con diversas políticas específicas en alguno de los campos de la PS, especialmente con respecto al tabaquismo, consumo de alcohol, actividad física y alimentación. Aún en su corta trayectoria las experiencias de promoción de la salud exitosas han mostrado que pueden hacer parte de las políticas de transformación progresiva de los sistemas de salud, buscando eficacia y aportes significativos a la calidad y esperanza de vida de la población.

Un balance preliminar

En la fase considerada del proceso y la experiencia de la PS, se han consolidado las definiciones proporcionadas por la Carta de Ottawa y por la OPS. Lo cual indica, como ya se planteó en la fase fundacional, que se ha consolidado la confluencia de paradigmas aunque las definiciones predominantes tienen un sello más claramente interpretativo, correspondiente a la teoría crítica y el construccionismo. 
La PS es quizás uno de los campos políticos, disciplinarios y profesionales donde se ha avanzado de manera sustancial en la confluencia y articulación de los paradigmas positivistas y no positivistas no sólo en sus bases ontológicas y epistemológicas, sino también en los aspectos metodológicos. En el presente, como resultado de la evaluación de las experiencias de PS, se recomienda la investigación "multiparadigmática" (49).

Se colige de lo anterior que la PS es un término polisémico y por tanto con pluralidad de significados. Así mismo, es un campo de conocimiento y práctica joven (40) que se ha desarrollado en un intenso proceso de diálogo y tensiones entre disciplinas y profesiones muy diversas (36, 55,56), apuntando a convertirse en un campo unitario con una teoría propia. Pero, es también claro que no cuenta aún con teoría subyacente completa, que permita establecer de manera acabada sus fronteras y darle unidad temática. Cabe mencionar que si bien es difícil identificar el cuerpo teórico unitario y propio de la PS, no ocurre lo mismo cuando se intenta definirla por lo que desea lograr, sus objetivos o por sus procesos. Buena parte de las definiciones de PS se centran en el proceso para obtener un resultado en salud. El proceso de construcción de la PS involucra múltiples aspectos: aproximaciones teóricas y conceptuales, experiencias, políticas públicas e institucionales, desarrollo y prueba de estrategias, conformación de escenarios de acción y de práctica, espacio de retos de investigación y, conjunto de métodos de evaluación.

Es quizá por esto que en PS historia y teoría se condensan en un breve período. Tanto la teoría como la práctica de la PS están haciendo aún su propia historia en la conformación de una disciplina unitaria

\section{REFERENCIAS}

1. Rootman I, Goodstadt M, Potvin L, Springett J. Un marco para la evaluación de la promoción de la salud. En: Organización Panamericana de la Salud-OMS. Evaluación de la promoción de la salud. Principios y perspectivas. Washington, DC: OPS; 2007. pp. 29-57.

2. Guba EG, Lincoln YS. Paradigmas en pugna en la investigación cualitativa. En: Denzin N, Lincoln I (eds.) Handbook of Qualitative Research, Cap. 6. London: Sage, 1994, pp.105-117

3. Colomer-Revuelta C, Álvarez-Dardet C. Promoción de la Salud y Cambio Social. Capítulo 3. En: Promoción de la Salud: concepto, estrategias métodos. Barcelona. MASSON S.A. 2001 
4. Mcqueen D.V. Perspectives on Health Promotion: Theory, Evidence, Practice and the Emergence of Complexity. Health Promotion International. Vol. 15, No. 2. Oxford University Press. 2000.

5. Rootman I, Goodstadt M, Potvin L, Springett J. Un marco para la evaluación de la promoción de la salud. En: Organización Panamericana de la Salud-OMS. Evaluación de la promoción de la salud. Principios y perspectivas. Washington, DC: OPS; 2007. pp. 29-57.

6. Winslow CEA. The untilled fields of health promotion. Science. 51:23, 1920.

7. Fee E, Morman ET. Doing history, making revolution: The aspirations of Henry E. Sigerist and George Rosen In: Porter D, Porter R (Ed.). Doctors, political and society: historical essays. Amsterdam, Atlanta: Editions Rodopi V.B.; 1993. p. 276).

8. Winslow CEA. The untilled fields of health promotion. Science. 51:23, 1920.

9. Sigerist HM. The university at the crossroads. New York: Henry Schuman, 1946.

10. Anderson R. Health promotion: an overview. Edinburgh, Scotish Health Education Group. European Monographs in Health Education Research, No. 6; 1984.

11. Stacey M. The Sociology of Health and Illness: Its Present State, Future Prospects and Potential for Health Research Sociology May 1978 12: 281-307.

12. McKweon T. Record RG, Brown RG. An interpretation of the modern rise of population in Europe. Population Studies. 1972; 27: 345.

13. McKeown T. The role of medicine: dream, mirage or nemesis. Oxford: Basil Blackwell; 1979.

14. McKeown T. Los orígenes de las enfermedades humanas. Barcelona: Editorial Crítica; 1990.

15. Laframboise HL. Health policy: breaking the problem down into more manageable segments. Canadian Medical Association Journal. 1973; 108: 388-393.

16. Porter D [Internet]. The History of Public Health: Current Themes and Approaches. Disponible en http://www.ep.liu.se/ej/hygiea/ra/002/paper.pdf Consultado Diciembre 2011).

17. Lalonde M. A new perspective on the health of Canadians: a working document. Ottawa, Canada Information, 1974.

18. Eslava-Castañeda JC. Repensando la Promoción de la Salud en el Sistema General de Seguridad Social en Salud. Rev. salud pública (Bogotá). Nov 2006; 8, suppl.2: 106-115).

19. Buck C. Después de Lalonde, hacia una generación de la salud. OPS Bol Epidemiol, 1986; 7(2):10-15).

20. Blum HL. Planing for health. 2nd Ed. New York: Human Science Press; 1981.

21. OMS/OPS. Promoción de la salud: Una antología. Washington: Publicación Científica No. 557. 1996. p. 312.

22. Antonovsky A, Health, stress and coping. San Francisco, Washington, London: LosseyBass Publishers; 1979.

23. Antonovsky A. The salutogenic model as a theory to guide health promotion. Health Promotion International @ Oxford University Press. 1996; 11, 1:11-18).

24. Green LW. National policy in the promotion of health. International Journal of health education. 1979; 22: 161-168.

25. Green LW. Current report: Office of health information, health promotion, Physical Fitness and Sports Medicine. Health education. 1980; 11 (2):28.

26. Green LW, Iverson DC. School health education. Annual Review of Public Health. 1982; 3:321-338.

27. Tannahill A. What is health promotion? Health education journal. 1985; 44:167-8.

28. OMS. Discussion document on the concept and principles of health promotion. Copenhagen, OMS Oficina Regional de Europa; 1984.

29. Nutbeam D. Health promotion glossary. Copenhagen, OMS Oficina Regional de Europa; 1985. (Document ICP/HBI 503 GO 4). 
30. Epp J. Achieving health for all. A framework for health promotion. Ottawa, Health and Welfare Canada; 1986.

31. Ottawa charter for health promotion. Health promotion. 1986; 1 (4): iii-v.

32. Cerqueira MT. Reflexión sobre la búsqueda de evidencia de efectividad de la promoción de la salud en las Américas. En: Organización Panamericana de la Salud-OMS. Evaluación de la promoción de la salud. Principios y perspectivas. Washington, DC: OPS; 2007. pp. 1-21.

33. Goodstadt MS. Health promotion: a conceptual integration. American Journal of health promotion. 1987; 1 (3): 58-63.

34. Kar SB (Ed.). Health promotion indicators and action. New York: Springer. 1989.

35. O'Donnell MP. Definition of health promotion. Part III. Expanding the definition. American Journal of Health Promotion. 1989; 3 (3):5.

36. Green LW, Kreuter MM. Health promotion planning: an educational and environmental approach. 2nd Ed. Mountain View, Mayfield; 1991.

37. Labonté R, Little S. Determinants of health: empowering strategies for nursing. Vancouver: Registered Nurses Associaton of British Columbia; 1992.

38. Kickbusch I. Introduction: tell me a story. In: Pederson, O' neill, Rootman (Ed). Health promotion in Canada. Canada: W. B. Saunders; 1994.

39. OPS/OMS. Orientaciones estratégicas y prioridades programáticas para el cuatrenio 1991-1994. Washington, D.C.; 1990.

40. Goodstadt MS, Hyndman B, McQueen DV, Potvin L, Rootman I, Springett J. Evaluación en la promoción de la salud: síntesis y recomendaciones. En: Organización Panamericana de la Salud-OMS. Evaluación de la promoción de la salud. Principios y perspectivas. Washington, DC: OPS; 2007. pp. 485-500.

41. Beattie A. Knowledge and control in health promotion: a taste case for social policy and social theory. In: Gabe J, Calnan M, Bury M. Eds. The sociology of health service. London: Routledge; 1991. pp. 162-202.

42. Ewles L, Simnett I. Promoting health: A practical Guide. 4th Ed. London: Baillièr Tindall; 1999.

43. Restrepo H, Málaga H. Promoción de la salud: Cómo construir vida saludable. Editorial Médica Panamericana; 2001.

44. Naidoo J, Wills J. Health Studies. An Introduction. New YorK: Palgrave; 2001.

45. De Salazar L. Municipios y comunidades saludables. El reto de la evaluación. Cali: Centro para el desarrollo y evaluación de tecnologías en Salud Pública- CEDETES; 2002.

46. Rootman I, Goodstadt M, Potvin L, Springett J. Un marco para la evaluación de la promoción de la salud. En: Organización Panamericana de la Salud-OMS. Evaluación de la promoción de la salud. Principios y perspectivas. Washington, DC: OPS; 2007. pp. 29-57.

47. Gendron S. Alianzas transformadoras entre los enfoques cualitativos y cuantitativos. En: Organización Panamericana de la Salud-OMS. Evaluación de la promoción de la salud. Principios y perspectivas. Washington, DC: OPS; 2007. pp. 119-131.

48. Rootman I, Goodstadt M, Potvin L, Springett J. Un marco para la evaluación de la promoción de la salud. En: Organización Panamericana de la Salud-OMS. Evaluación de la promoción de la salud. Principios y perspectivas. Washington, DC: OPS; 2007. pp. 29-57.

49. Gendron S. Alianzas transformadoras entre los enfoques cualitativos y cuantitativos. En: Organización Panamericana de la Salud-OMS. Evaluación de la promoción de la salud. Principios y perspectivas. Washington, DC: OPS; 2007. pp. 119-131.

50. OPS-OMS. Evaluación de la Promoción de la Salud. Principios y perspectivas. Washington, D.C: OPS; 2007. p. 29-30.

51. Perry $C L$, Jessor $R$, The concept of health promotion and the prevention of adolescent drug abuse. Health education quarterly. 1985; 12 (2): 169-184. 
52. Ruttten A. Evaluación de las políticas públicas saludables en contextos comunitarios y regionales. En: Organización Panamericana de la Salud-OMS. Evaluación de la promoción de la salud. Principios y perspectivas. Washington, DC: OPS; 2007. pp. 327-347.

53. Warren R. Rootman I, Wilson R. Evaluación de las políticas de promoción de la salud a nivel nacional: la experiencia canadiense. En: Organización Panamericana de la Salud-OMS. Evaluación de la promoción de la salud. Principios y perspectivas. Washington, DC: OPS; 2007. pp. 369-383.

54. Frankish CJ, Green LW, Ratner PA, Chomik T, Larsen C. Evaluación del impacto en la salud como una herramienta para la promoción de la salud y la salud poblacional. En: Organización Panamericana de la Salud-OMS. Evaluación de la promoción de la salud. Principios y perspectivas. Washington, DC: OPS; 2007. pp. 385-413.

55. Mcqueen DV. The search for theory in health behaviour and health promotion. Health promotion international. 1996; 11 (1):27-32.

56. Dean K. Researching population health. New directions. In: Dean K (Ed). Population health research: linking theory and methods. London: Sage; 1993. p. 227-237. 